\title{
GENERAL $n$-DIMENSIONAL TAUBERIAN PROBLEMS WITH APPLICATION TO THE LAPLACE- AND STIELTJES TRANSFORMS
}

\author{
LENNART FRENNEMO
}

\begin{abstract}
A general theorem on the closure of translates in certain weighted spaces in $R^{n}$ is proved and as a consequence a general $n$-dimensional Tauberian theorem. This is applied to the $n$-dimensional Laplace transform and to the one-dimensional Stieltjes- and Weierstrass transforms.
\end{abstract}

\section{Introduction}

Let $K, \phi$ and $\psi$ be functions from $R^{n}$ to $R$ which belong to some specific classes of functions which will be defined later in the text.

Suppose that

$$
K * \phi(x) \sim K * \psi(x), \quad x \rightarrow+\infty .
$$

Under certain restrictions on $\psi$ and with a Tauberian condition on $\phi$ we will show that

$$
\phi(x) \sim \psi(x), \quad x \rightarrow+\infty .
$$

Here

$$
K * \phi(x)=\int K(x-u) \phi(u) d u,
$$

where we let an unspecified region of integration be $R^{n}$ throughout the text.

By $x=\left(x_{1}, x_{2}, \ldots, x_{n}\right) \rightarrow+\infty$ we mean that $x_{k} \rightarrow+\infty, k=1,2, \ldots, n$, and by (0.2) we mean that

$$
\phi(x)=\psi(x)+o(\psi(x)) \quad \text { when } \quad x \rightarrow+\infty,
$$

with a corresponding interpretation for (0.1).

The class of kernels considered here will be chosen so that it includes a wide variety of well-known transformation kernels. As specific examples we

Received December 15, 1999. 
apply the general results to the $n$-dimensional Laplace transform and the onedimensional Stieltjes- and Weierstrass transforms.

Problems of this kind for the one dimensional Laplace transform has been treated earlier by the author in [7].

The method used depends on an $n$-dimensional analogue of a theorem on the closure of the span of translates of the transformation kernels in a certain weighted space. One-dimensional closure theorems of this kind was first proved by Nyman [14] and Korenblum [11]. The methods used in this paper are developments of ideas used by the author in ([5], [6], [7]).

\section{Preliminaries}

We will use the following notations beside the ones used in the introduction.

If

$$
x=\left(x_{1}, x_{2}, \ldots, x_{n}\right) \in R^{n}, \quad y=\left(y_{1}, y_{2}, \ldots, y_{n}\right) \in R^{n}
$$

then

$$
x \leq y \quad \text { if } \quad x_{k} \leq y_{k} \quad \text { for all } \quad k=1,2, \ldots, n,
$$

with a corresponding meaning for $x<y$.

We also let $R_{+}^{n}$ be all $x \in R^{n}$ such that $x \geq \mathbf{0}=(0,0, \ldots, 0)$.

Furthermore, we let

$$
\begin{aligned}
x \cdot y & =\sum_{k=1}^{n} x_{k} y_{k},|x|=\sqrt{x \cdot x}, x \otimes y=\left(x_{1} y_{1}, x_{2} y_{2}, \ldots, x_{n} y_{n}\right), \\
\delta x & =\left(\delta x_{1}, \delta x_{2}, \ldots, \delta x_{n}\right) \text { if } \delta \text { is a real number, } \\
\|x\| & =\sum_{k=1}^{n} \max \left(0, x_{k}\right),\left(\text { which is a pseudonorm in } R^{n} \text { but a norm in } R_{+}^{n}\right), \\
\exp x & =\left(\exp x_{1}, \exp x_{2}, \ldots, \exp x_{n}\right), \quad \mathbf{1}=(1,1, \ldots, 1) . \\
\text { If } x>\mathbf{0} \text { then } &
\end{aligned}
$$

$$
\begin{aligned}
\ln x & =\left(\ln x_{1}, \ln x_{2}, \ldots, \ln x_{n}\right), x^{y}=\exp (y \cdot \ln x), \\
\frac{x}{y} & =\left(\frac{x_{1}}{y_{1}}, \frac{x_{2}}{y_{2}}, \ldots, \frac{x_{n}}{y_{n}}\right) .
\end{aligned}
$$

We use standard notations for the Fouriertransform, thus

$$
\hat{K}(x)=\int \exp (-i x \cdot t) K(t) d t .
$$


We also introduce weight-functions $p$ defined in $R^{n}$ such that

$$
\begin{aligned}
& p(x) \geq p(0)=1 \\
& p(x+y) \leq p(x) p(y) \\
& p(r x) \geq p(x) \quad \text { if } r \text { real and } r \geq 1
\end{aligned}
$$

In this paper we use weight-functions $p$ of the form

$$
p(x)=(1+|x|)^{\mu} \exp (\|m \otimes x\|), \text { where } \mu \in R_{+} \text {and } m \in R_{+}^{n} .
$$

We call a function $p$ from $R^{n}$ to $R$ non-decreasing if

$$
p(x) \leq p(y) \quad \text { when } x \leq y .
$$

Definition 1.1. For any weight-function $p$ we let $L^{1}(p)$ consist of all measurable functions $H$ such that

$$
\|H\|_{p}^{1}=\int|H(-x)| p(x) d x<\infty
$$

We also let $L^{\infty}(p)$ denote the space of all measurable functions $\phi$ such that

$$
\|\phi\|_{p}^{\infty}=\operatorname{ess} \sup _{-\infty<x<\infty} \frac{|\phi(x)|}{p(x)}<\infty .
$$

We see that $L^{1}(p)$ is a Banachspace under this norm with $L^{\infty}(p)$ as its dual space, which means that any bounded linear functional on $L^{1}(p)$ is of the form

$$
K \rightarrow \int K(-x) \phi(x) d x
$$

for some function $\phi \in L^{\infty}(p)$. (Cf. e.g. [16] p. 136).

For convenience we let $C$ stand for positive constants not necessarily the same each time.

\section{Some general Tauberian Theorems}

We first introduce the class of kernels considered.

Definition 2.1. By $E(\alpha, \beta, M)$ we denote all integrable functions $K$ defined in $R^{n}$ such that:

$1^{0} \hat{K}(t) \neq 0$ for all $t \in R^{n}$

$2^{0}$ The function $g$ defined by $g(t)=\hat{K}(t)^{-1}$ can be analytically continued in a region $-\alpha<\operatorname{Im} t<\beta$ for some $\alpha, \beta>0$ 
$3^{0}$ This function $g$ satisfies an inequality

$$
|g(t)| \leq C \exp (M(x))
$$

for all $t=x+i y$ such that $-\alpha<y<\beta$ and for some function $M$ from $R^{n}$ to $R_{+}$.

THeOREM 2.2. Let $p$ be a weight-function of the form

$$
\begin{aligned}
& \quad p(x)=(1+|x|)^{\mu} \exp (\|m \otimes x\|), x \in R^{n} \text {, for some } \mu \text { in } R_{+} \text {and } m \text { in } R_{+}^{n} . \\
& \qquad \text { If } K \in L^{1}(p) \cap E(\alpha, \beta, M) \text { with } \alpha>m \text { and if } M(x)=o\left(\sum_{k=1}^{n} \exp \left(\pi \frac{\left|x_{k}\right|}{\alpha_{k}}\right)\right) \\
& \text { when }|x| \rightarrow \infty \text {, then the span of translates of } K \text { is dense in } L^{1}(p) \text {. }
\end{aligned}
$$

Proof. We will prove that for any $\phi \in L^{\infty}(p)$ and any $K$ which satisfies the condition above then

$$
K * \phi(x)=0 \quad \text { for all } \quad x \in R^{n}
$$

implies that

$$
\phi(x)=0 \quad \text { a.e. in } R^{n} .
$$

For any real $\varepsilon>0$ such that $\alpha>m+\frac{3 \varepsilon}{\pi} \alpha$ and $\beta-\frac{2 \varepsilon}{\pi} \alpha>0$, and for any $\omega \in R^{n}$ consider the function

$$
Q(x)=\frac{1}{(2 \pi)^{n}} \int \exp (i x \cdot u) h(u-\omega) g(u) d u
$$

where

$h(u)=\exp \left[-A \cdot \exp \left(\pi \frac{u}{\alpha}+i\left(\frac{\pi}{2}-2 \varepsilon\right) \mathbf{1}\right)-A \cdot \exp \left(-\pi \frac{u}{\alpha}-i\left(\frac{\pi}{2}-2 \varepsilon\right) \mathbf{1}\right)\right]$

with an $A \in R_{+}^{n}$ such that $\cos \left(\frac{\pi}{2}-\varepsilon\right) A=\mathbf{1}$.

In (2.2) we now make the substitution

$$
u=t+i s \quad \text { where } \quad-\alpha+\frac{2 \epsilon}{\pi} \alpha<s<\frac{2 \varepsilon}{\pi} \alpha,
$$

and after a translation of the region of integration we obtain that

$$
Q(-x)=\frac{1}{(2 \pi)^{n}} \exp (s \cdot x) \int \exp (-i x \cdot t) h(t-\omega+i s) g(t+i s) d t
$$

If now $s=\left(s_{1}, s_{2}, \ldots, s_{n}\right)$ is chosen so that

$$
s_{k}=-\alpha_{k}+\frac{3 \varepsilon}{\pi} \alpha_{k} \quad \text { when } \quad x_{k}>0
$$


and

$$
s_{k}=+\varepsilon \frac{\alpha_{k}}{\pi} \quad \text { when } \quad x_{k} \leq 0
$$

then we can see that there exists a positive number $\delta$ such that

$$
|Q(-x) p(x)| \leq C \exp (-\delta|x|) \quad \text { for all } \quad x \in R^{n} .
$$

Hence $Q \in L^{1}(p)$.

If

$$
K * \phi=\psi
$$

then clearly $\psi \in L^{\infty}(p)$ and

$$
Q *(K * \phi)=Q * \psi .
$$

The conditions above are enough to prove that

$$
Q *(K * \phi)=(Q * K) * \phi .
$$

The method above to prove formulas (2.4) and (2.5) can also be used to prove that if $h$ is defined as in (2.3) then $h=\hat{H}$ for some function $H \in L^{1}(p)$. Now

$$
Q * K(u)=H(u) \exp (i \omega \cdot u),
$$

which follows from the fact that

$$
\hat{Q}(u)=h(u-\omega) g(u)
$$

and hence

$$
(Q * K) \hat{(u)}=\hat{Q}(u) \hat{K}(u)=h(u-\omega) .
$$

By use of (2.1), (2.7) and (2.8) we have that

$$
\int \exp (-i \omega \cdot u) H(-u) \phi(u+x) d u=0 \quad \text { for all } \quad \omega, x \in R^{n}
$$

The uniqueness of the Fouriertransform now implies that

$$
H(-u) \phi(u+x)=0 \text { a.e. in } u \text { for any } x \in R^{n},
$$

and since $H$ is non-trivial, we have finally proved that

$$
\phi(u)=0 \text { a.e. in } R^{n} .
$$

It now follows from the Hahn-Banach theorem that the span of translates of $K$ is dense in $L^{1}(p)$. (Cf. [16] p. 114). 
Remark 2.3. In thesis 1950 Nyman [14] gave, in the one-dimensional case, the necessary and sufficient conditions for the span of translates of a kernel $K \in L^{1}(p)$ to be dense in $L^{1}(p)$, if $p(x)=\exp (\alpha x)$ for $x \geq 0$ and $p(x)=\exp (-\beta x)$ for $x<0$. He proved that $\hat{K}(t) \neq 0$ in the closed strip $-\alpha \leq \operatorname{Im} t \leq \beta$ and $K \in E(\alpha, \beta, M)$ with $M(x)=o\left(\exp \frac{\pi|x|}{\alpha+\beta}\right)$ when $x \rightarrow \pm \infty$, are the appropriate conditions.

Essentially the same result was proved by Korenblum [11] in 1958.

In this connection it may also be noted that a well-known result by Levinson [12], also in the one-dimensional case, shows that if $m>\alpha$ then

$$
H(x)=O(\exp (-m x)) \text { and } \hat{H}(x)=O\left(\exp \left(-\exp \left(\frac{\pi}{\alpha} x\right)\right)\right), x \rightarrow+\infty
$$

implies that $H(x)=0$ a.e.

THEOREM 2.4. Let $K, \phi$ and $\psi$ be functions from $R^{n}$ to $R$ such that $K$ is non-negative and fulfills the conditions of Theorem 1 with $p(x)=(1+$ $|x|) \exp (\|m \otimes x\|)$. Furthermore, let $\psi$ be positive and non-decreasing and let $\phi(x)$ and $\psi(x)$ be bounded when $\|x\|$ is bounded.

If now

$$
K * \phi(x) \sim K * \psi(x), \quad x \rightarrow+\infty
$$

and also

$$
K * \phi(x)=O(K * \psi(x)) \text { when }\|x\| \rightarrow+\infty
$$

and if for any real $\delta>0$ there exist an $X$ in $R$ such that

$$
\psi(x+y) \leq(1+\delta) \exp (m \cdot y) \psi(x) \text { when }\|x\| \geq X, y \geq 0 .
$$

and if

$$
\lim _{h \rightarrow 0+} \liminf _{\|x\| \rightarrow \infty} \inf _{x \leq y \leq x+h}\left(\frac{\phi(y)-\phi(x)}{\psi(x)}\right)=0
$$

then

$$
\phi(x) \sim \psi(x), \quad x \rightarrow+\infty .
$$

Proof. As a first step we will prove that

$$
|\phi(x)| \leq C(1+\psi(x)) \text { for all } x \in R^{n} .
$$

In proving this we use that

$$
\psi(x+u) \leq C \exp (\|m \otimes u\|)(1+\psi(x)) \quad \text { for any } \quad u, x \in R^{n} .
$$


This follows since $\psi$ is non-decreasing and hence

$\psi(x+u) \leq \psi(x+y) \quad$ if $\quad y=\left(\left\|u_{1}\right\|,\left\|u_{2}\right\|, \ldots,\left\|u_{n}\right\|\right) \quad$ for any $x, u \in R^{n}$ and then by (2.12)

$\psi(x+u) \leq C \exp (\|m \otimes u\|) \psi(x)$ for any $u \in R^{n}$ if $\|x\|$ is large enough.

Since $\psi(x)$ is bounded when $\|x\|$ is bounded, we easily get (2.15).

We now start using (2.13) and the fact that $\phi(x)$ is bounded when $\|x\|$ is bounded to see that

(2.16) $\phi(y)-\phi(x) \geq-C(1+\psi(x)), \quad x \leq y \leq x+21$ for all $x \in R^{n}$.

If $x \leq y \leq x+\mathbf{1}$ then

$K * \phi(y+\mathbf{1})-K * \phi(x)=\int K(-u)(\phi(u+y+\mathbf{1})-\phi(u+x)) d u=I_{1}+I_{2}$

where $I_{1}$ is the integral taken over all $S=\left\{u \in R^{n}:-\mathbf{1} \leq u \leq \mathbf{0}\right\}$ and $I_{2}$ is the integral taken over the rest of $R^{n}$.

By (2.15) and (2.16) we see that

$$
I_{2} \geq-C(1+\psi(x))
$$

and hence by (2.11) and (2.15) we have that

$$
I_{1} \leq K * \phi(y+\mathbf{1})-K * \phi(x)+C(1+\psi(x)) \leq C(1+\psi(x))
$$

if $\|x\|$ is large enough.

When $u \in S$ we write

$$
\begin{aligned}
& \phi(u+y+\mathbf{1})-\phi(u+x) \\
& \quad=\phi(u+y+\mathbf{1})-\phi(y)+\phi(x)-\phi(u+x)+\phi(y)-\phi(x) \\
& \geq-C(1+\psi(x))+\phi(y)-\phi(x)
\end{aligned}
$$

and see that

$$
I_{1} \geq(-C(1+\psi(x))+\phi(y)-\phi(x)) \int_{S} K(-u) d u .
$$

Since $\phi(x)$ is bounded if $\|x\|$ is bounded we have from (2.17) that $\phi(y)-\phi(x) \leq C(1+\psi(x))$ for all $x, y \in R^{n}$ such that $x \leq y \leq x+\mathbf{1}$. 
We combine this inequality with (2.16) and see that

$$
|\phi(u+x)-\phi(x)| \leq C(1+\psi(x)), \quad \mathbf{0} \leq u \leq \mathbf{1} \text { for any } x \in R^{n} .
$$

Hence for any $u \geq 0$ and any $u \leq 0$ we have by (2.15) that

$|\phi(u+x)-\phi(x)| \leq C(1+|u|) \exp (\|m \otimes u\|)(1+\psi(x)) \leq C p(u)(1+\psi(x))$.

For any other value of $u$ we let

$$
\begin{aligned}
x_{0}=x, x_{1}= & x+\left(u_{1}, 0,0, \ldots\right), \\
& x_{2}=x_{1}+\left(0, u_{2}, 0, \ldots\right), x_{3}=x_{2}+\left(0,0, u_{3}, 0, \ldots\right), \ldots
\end{aligned}
$$

and in this case we see after some calculations using (2.18) and (2.15) that

$$
\begin{aligned}
& |\phi(u+x)-\phi(x)|=\left|\sum_{q=1}^{n}\left(\phi\left(x_{q}\right)-\phi\left(x_{q-1}\right)\right)\right| \\
& \leq C \sum_{q=1}^{n}\left(1+\left|u_{q}\right|\right) \exp \left(\left\|m_{q} u_{q}\right\|\right)\left(1+\psi\left(x_{q-1}\right)\right) \leq C p(u)(1+\psi(x))
\end{aligned}
$$

Hence

(2.19) $\quad|\phi(u+x)-\phi(x)| \leq C p(u)(1+\psi(x))$ for all $u$ and $x$ in $R^{n}$.

Now since $K \in L^{1}(p)$

$$
\begin{aligned}
&\left|K * \phi(x)-\phi(x) \int K(-u) d u\right|=\left|\int K(-u)(\phi(u+x)-\phi(x)) d u\right| \\
& \leq C(1+\psi(x)) \int K(-u) p(u) d u \leq C(1+\psi(x)) .
\end{aligned}
$$

Finally we use (2.11) and the fact that $\phi(x)$ is bounded when $\|x\|$ is bounded to see that

$$
|\phi(x)| \leq C(1+\psi(x)) \text { for all } x \in R^{n} .
$$

Hence the first step of the proof is completed.

For any function $H \in L^{1}(p)$ and for any $\varepsilon>0$ we now can, using Theorem 2.2, find a finite linear combination $K_{\varepsilon}$ of translates of $K$,

$$
K_{\varepsilon}(x)=\sum_{k=1}^{m} a_{k} K\left(x-\lambda_{k}\right),
$$


such that

$$
\left\|K_{\varepsilon}-H\right\|_{p}^{1}<\varepsilon
$$

We write

$$
H * \phi=H * \psi+\left(H-K_{\varepsilon}\right) *(\phi-\psi)+K_{\varepsilon} *(\phi-\psi) \text {. }
$$

By (2.10) and (2.12) we see that

$$
K_{\varepsilon} *(\phi-\psi)=o(1) K_{\varepsilon} * \psi(x)=o(\psi(x)), \quad x \rightarrow+\infty .
$$

By (2.20) we have that

$$
\left(H-K_{\varepsilon}\right) *(\phi-\psi)(x)=O(1)\left(\left|H-K_{\varepsilon}\right| *(1+\psi(x)), \quad x \rightarrow+\infty,\right.
$$

and hence by (2.15) we have that

$$
\begin{aligned}
\left(H-K_{\varepsilon}\right) *(\phi-\psi)(x) & =O(1) \int\left|H(-u)-K_{\varepsilon}(-u)\right|(1+\psi(u+x)) d u \\
& =O(1)(1+\psi(x)) \int\left|H(-u)-K_{\varepsilon}(-u)\right| p(u) d u \\
& =O(1)(1+\psi(x))\left\|H-K_{\varepsilon}\right\|_{p}^{1}, \quad x \rightarrow+\infty
\end{aligned}
$$

Since $\varepsilon$ is arbitrary, it follows that

$$
H * \phi(x)=H * \psi(x)+o(\psi(x)), \quad x \rightarrow+\infty .
$$

For any positive real number $h$ we now let $H$ be the characteristic function on the set $S_{h}=\left\{u \in R^{n}:-h \mathbf{1} \leq u \leq \mathbf{0}\right\}$ multiplied by $h^{-n}$. Then by (2.21)

$$
h^{-n} \int_{S_{h}} \phi(x-u) d u=h^{-n} \int_{S_{h}} \psi(x-u) d u+o(\psi(x)), \quad x \rightarrow+\infty .
$$

We divide this expression with $\psi(x)$ and see that

$$
\begin{aligned}
\frac{\phi(x)}{\psi(x)} & =h^{-n} \int_{S_{h}} \frac{\phi(x)}{\psi(x)} d u \\
& =h^{-n} \int_{S_{h}} \frac{\phi(x)-\phi(x-u)}{\psi(x)} d u+h^{-n} \int_{S_{h}} \frac{\phi(x-u)}{\psi(x)} d u \\
& =h^{-n} \int_{S_{h}} \frac{\phi(x)-\phi(x-u)}{\psi(x)} d u+h^{-n} \int_{S_{h}} \frac{\psi(x-u)}{\psi(x)} d u+o(1), x \rightarrow \infty .
\end{aligned}
$$


By use of (2.12) and (2.13) we see that if $h$ is small enough then to any real $\varepsilon>0$ there exists an $x_{1} \in R^{n}$ such that

$$
\frac{\phi(x)}{\psi(x)}<1+\varepsilon \quad \text { if } \quad x \geq x_{1} .
$$

If on the other hand, $H$ is the characteristic function on $D_{h}=\left\{u \in R^{n}: 0 \leq\right.$ $u \leq h \mathbf{1}\}$ multiplied by $h^{-n}$ we can in an analogous way prove that there exists an $x_{2} \in R^{n}$ so that

$$
\frac{\phi(x)}{\psi(x)}>1-\varepsilon \quad \text { if } \quad x \geq x_{2} .
$$

Hence

$$
\phi(x) \sim \psi(x), \quad x \rightarrow+\infty,
$$

and we have proved Theorem 2.4.

\section{Results for the $\boldsymbol{n}$-dimensional Laplace transform}

We suppose that $\alpha$ and $\beta$ are real functions of bounded variation defined in $R_{+}^{n}$. We also suppose that

$$
\alpha(t)=\beta(t)=0 \quad \text { if any } \quad t_{k}=0, k=1,2, \ldots, n .
$$

This means that $\alpha$ and $\beta$ belong to an $n$-dimensional analogue of class $V_{0}$ in [1].

We use the following notations for the corresponding Laplace transforms:

$$
\begin{aligned}
& F(s)=\int_{R_{+}^{n}} \exp (-s \cdot t) d \alpha(t), \quad s>0, \\
& G(s)=\int_{R_{+}^{n}} \exp (-s \cdot t) d \beta(t), \quad s>0,
\end{aligned}
$$

where we suppose that the integrals are boundedly convergent for any $s>0$ (cf. [1]).

THEOREM 3.1. Let $\beta$ be positive and non-decreasing and suppose that

$$
F(s) \sim G(s), \quad s \rightarrow \mathbf{0}+,
$$

and that

$$
F(s)=O(G(s)) \quad \text { when } \min _{k=1,2, \ldots, n} s_{k} \rightarrow 0+.
$$


If for any real number $\delta>0$ there exist an $m \in R_{+}^{n}$ and a positive real number $T$ such that

$$
\beta(r \otimes t) \leq(1+\delta) r^{m} \beta(t), \quad r \geq \mathbf{1}, \quad\|t\| \geq T
$$

and if

$$
\lim _{\lambda \rightarrow 1+} \liminf _{\|x\| \rightarrow \infty} \inf _{x \leq t \leq \lambda x}\left(\frac{\alpha(t)-\alpha(x)}{\beta(x)}\right)=0
$$

then

$$
\alpha(t) \sim \beta(t), \quad t \rightarrow \infty
$$

Proof. We make a partial integration in (3.1) and obtain that

$$
F(s)=s^{1} \int_{R_{+}^{n}} \exp (-s \cdot t) \alpha(t) d t, \quad s>0,
$$

where the integral is absolutely convergent (cf. [1]). We do the same in (3.2) and get a corresponding result for $G(s)$.

We now make the substitutions

$$
s=\exp (-x) \text { and } t=\exp u
$$

and let

$$
\phi(x)=\alpha(\exp x) \quad \text { and } \quad \psi(x)=\beta(\exp x) .
$$

In this way (3.3) is transformed into (2.10), that is

$$
K * \phi(x) \sim K * \psi(x), \quad x \rightarrow+\infty,
$$

where

$$
K(x)=\exp (-\mathbf{1} \cdot \exp (-x)-\mathbf{1} \cdot x)
$$

and

$$
\hat{K}(t)=\prod_{k=1}^{n} \Gamma\left(1+i t_{k}\right), \quad t=\left(t_{1}, t_{2}, \ldots, t_{n}\right) .
$$

Hence $K$ fulfills the conditions required in Theorem 2.4. That $K \in E(\alpha, \beta, M)$ for any $\alpha>0$ and properly chosen $\beta$ and $M$ follows from Stirlings formula (cf. e.g. [6] p. 231). It is also easy to see that the other conditions of Theorem 2.4 are fulfilled since (2.11), (2.12) and (2.13) are consequences of (3.4), (3.5) and (3.6) respectively. Now the conclusion (3.7) follows from (2.14) and hence Theorem 3.1 is a consequence of Theorem 2.4. 
We also have the following corollary to Theorem 3.1:

Corollary 3.2. Let $m \in R_{+}^{n}$ and let $A$ be a positive real number. Suppose that

$$
F(s) \sim A s^{-m}, \quad s \rightarrow \mathbf{0}+
$$

and that

$$
F(s)=O\left(s^{-m}\right) \quad \text { when } \quad \min _{k=1,2, \ldots, n} s_{k} \rightarrow 0+.
$$

Furthermore, suppose that

$$
\lim _{\lambda \rightarrow 1+\|x\| \rightarrow \infty} \liminf _{x \leq t \leq \lambda x}\left(\frac{\alpha(t)-\alpha(x)}{x^{m}}\right)=0,
$$

then

$$
\alpha(t) \sim \frac{A t^{m}}{\prod_{k=1}^{m} \Gamma\left(1+m_{k}\right)}, \quad t \rightarrow \infty
$$

Proof. In Theorem 3.1 we let

$$
\beta(t)=\frac{A t^{m}}{\prod_{k=1}^{n} \Gamma\left(1+m_{k}\right)}, \quad \text { if } \quad t>\mathbf{0},
$$

and

$$
\beta(t)=0 \quad \text { if any } \quad t_{k}=0, k=1,2, \ldots, n .
$$

In this case,

$$
G(s)=A s^{-m}, \quad s>\mathbf{0},
$$

and the corollary follows from this.

Remark 3.3. From this corollary it follows that Theorem 3.1 is an $n$-dimensional generalisation of the classical Hardy-Littlewood-Karamata theorem for the Laplace transform (cf. [8], [10], [13]). If $n=1$ condition (3.4) is included in (3.3) and hence the classical one-dimensional results are included in Theorem 3.1.

The two-dimensional case of this corollary was first treated by Delange [4] in the special case when $m=\mathbf{0}$. He then used Tauberian conditions strong enough to imply bounded convergence of the Laplace transform. More recently the multidimensional case of Corollary 3.2 has been treated among others by Celidze ([2], [3]), Stadtmüller and Trautner [17] and Omey and Willekens [15]. They also use stronger conditions than ours which imply both bounded convergence and condition (3.9). 
In Theorem 3.1 we could equally well let $\alpha$ and $\beta$ be measures on $R_{+}^{n}$. We just have to replace $\alpha(t)$ and $\beta(t)$ in (3.5), (3.6) and (3.7) with $\int_{0 \leq s \leq t} d \alpha(s)$ and $\int_{0 \leq s \leq t} d \beta(s)$ respectively. (Cf. e.g. [5] p. 48).

\section{Consequences for the one-dimensional Stieltjes- and Weierstrass transforms}

Suppose that $\alpha$ and $\beta$ are real-valued functions of bounded variation defined in $R_{+}$with $\alpha(0)=\beta(0)=0$. For the Stieltjes transform we give the following example as a consequence of Theorem 2.4.

Theorem 4.1. Suppose that $\beta$ is positive and non-decreasing and that

$$
F(s)=\int_{0}^{\infty} \frac{d \alpha(t)}{s+t} \sim G(s)=\int_{0}^{\infty} \frac{d \beta(t)}{s+t}, \quad s \rightarrow \infty
$$

and also that for any real number $\delta$ there exist a real number $m, 0 \leq m<1$, and a real number $T$ such that

$$
\beta(r t) \leq(1+\delta) r^{m} \beta(t), \quad r \geq 1, t \geq T .
$$

Furthermore, suppose that

$$
\lim _{\lambda \rightarrow 1+} \liminf _{x \rightarrow \infty} \inf _{x \leq t \leq \lambda x}\left(\frac{\alpha(t)-\alpha(x)}{\beta(x)}\right)=0,
$$

then

$$
\alpha(t) \sim \beta(t), \quad t \rightarrow \infty
$$

Proof. After a partial integration in (4.1), we have that

$$
F(s)=\int_{0}^{\infty} \frac{\alpha(t) d t}{(s+t)^{2}}
$$

and with a corresponding result for $G(s)$.

We now make the substitutions

$$
s=\exp x \quad \text { and } \quad t=\exp y
$$

and thus transform the problem into Theorem (2.4) with $K(x)=\left(\exp \left(\frac{x}{2}\right)+\exp \left(-\frac{x}{2}\right)\right)^{-2}, \phi(x)=\alpha(\exp x)$ and $\psi(x)=\beta(\exp x)$.

In this case

$$
s F(s)=K * \phi(x) \quad \text { and } \quad \hat{K}(x)=2 \pi x(\exp (\pi x)-\exp (-\pi x))^{-1} .
$$


We omit the details of the proof.

Since

$\int_{0}^{\infty} \frac{t^{\alpha}}{(s+t)^{2}} d t=\frac{\Gamma(1-\alpha) \Gamma(1+\alpha)}{s^{1-\alpha}},-1<\alpha<1$, (cf. e.g. [18], p. 184),

we have the following corollary if we let

$$
\beta(t)=A \frac{t^{1-\gamma}}{\Gamma(\gamma) \Gamma(2-\gamma)}, \quad 0<\gamma \leq 1,
$$

in Theorem 4.1.

Corollary 4.2. If $0<\gamma \leq 1$ and if for any positive number $A$

$$
F(s) \sim A s^{-\gamma}, \quad s \rightarrow \infty
$$

and if also

$$
\lim _{\lambda \rightarrow 1+} \liminf _{x \rightarrow \infty} \inf _{x \leq t \leq \lambda x}\left(\frac{\alpha(t)-\alpha(x)}{x^{1-\gamma}}\right)=0,
$$

then

$$
\alpha(t) \sim A \frac{t^{1-\gamma}}{\Gamma(\gamma) \Gamma(2-\gamma)}, \quad t \rightarrow \infty
$$

This is a classical Tauberian theorem for the Stieltjes transform in a general version.

We finally give an example for the Weierstrass transform. The Weierstrass transform is the convolution transform

$$
f(x)=\int K(x-t) \phi(t) d t
$$

where

$$
K(x)=(4 \pi)^{-1 / 2} \exp \left(-\frac{x^{2}}{4}\right), \quad \text { (cf. [9] p. 174), }
$$

and we just give the following corollary to Theorem 2.4.

Corollary 4.3. Suppose that for any positive number $A$ and positive natural number $n$

$$
f(x) \sim A x^{n}, \quad x \rightarrow+\infty,
$$

and that $\phi(x)$ is bounded when $x$ is bounded above. If also

$$
\lim _{h \rightarrow 0+} \liminf _{x \rightarrow+\infty} \inf _{x \leq y \leq x+h}\left(\frac{\phi(y)-\phi(x)}{x^{n}}\right)=0
$$


then

$$
\phi(x) \sim A x^{n}, \quad x \rightarrow+\infty
$$

Proof. Suppose that

$$
H_{n}(x)=(-1)^{n} \exp \left(x^{2}\right) D^{n} \exp \left(-x^{2}\right), \quad n=0,1,2, \ldots
$$

are the Hermite polynomials.

We use the one-dimensional analogue of Theorem 2.4 with the Weierstrass kernel $K$ and with

$$
\psi(x)=H_{n}\left(\frac{x}{2}\right) .
$$

The result now follows from the fact that

$$
H_{n}\left(\frac{x}{2}\right) \sim x^{n}, \quad x \rightarrow+\infty
$$

and that

$$
\int K(x-t) H_{n}\left(\frac{t}{2}\right) d t=x^{n}, \quad \text { (cf. [9] p. 178). }
$$

The other conditions of Theorem 2.4 are clearly fulfilled.

\section{REFERENCES}

1. Bernstein, D. L., The double Laplace integral, Duke Math. J. 8 (1941), 460-496.

2. Celidze, É. V., Generalization of certain theorems of Karamata to the case of two variables (Russian), Izv. Vyssh. Ucehbn. Zaved. Mat. 1201972 no. 5 (1972), 109-115.

3. Celidze, É. V., A Tauberian theorem for double integrals (Russian), Trudy Tbiliss. Univ. 225 (1981), 81-95.

4. Delange, H., Théorèmes Tauberiens pour les series multiples de Dirichlet et les intégrales multiples de Laplace, Ann. Sci. École Norm. Sup. (3) 70 (1953), 51-103.

5. Frennemo, L., Tauberian problems for the n-dimensional Laplace transform I, Math. Scand. 19 (1966), 41-53.

6. Frennemo, L., Tauberian problems for the n-dimensional Laplace transform II, Math. Scand. 20 (1967), 225-239.

7. Frennemo, L., A generalisation of Littlewood's Tauberian theorem for the Laplace transform, Preprint, Department of Mathematics, 41296 Göteborg.

8. Hardy, G. H. and Littlewood, J. E., Notes on the theory of series XI. On Tauberian theorems, Proc. London Math. Soc. (2) 30 (1929), 23-37.

9. Hirschman, I. I. and Widder, D. V., The convolution transform, Princeton University Press, 1955.

10. Karamata, J., Neuer Beweis und Verallgemeinerungen einiger Tauberian-Sätze, Math. Z. 33 (1931), 294-299.

11. Korenblum, B., A generalisation of Wiener's Tauberian theorem and harmonic analysis of rapidly increasing functions (Russian), Trudy Moskov. Mat. Obshch. 7 (1958), 121-148.

12. Levinson, N., Restrictions imposed by certain functions on their Fourier transforms, Duke Math. J. 6 (1940), 722-731. 
13. Littlewood, J. E., The converse of Abel's theorem, Proc. London Math. Soc. (2) (1910), 434448.

14. Nyman, B., On the one-dimensional translation group and semi-group in certain function spaces, dissertation, Uppsala, 1950.

15. Omey, E. and Willekens, E., Abelian and Tauberian theorems for the Laplace transform of functions in several variables, J. Multivariate Anal. 30 (1989), no. 2, 292-306.

16. Rudin, W., Real and Complex Analysis, Mc Graw-Hill 1974.

17. Stadtmüller, U. and Trautner, R., Tauberian theorems for Laplace transforms in dimension $D>1$, J. Reine Angew. Math. 323 (1981), 127-138.

18. Widder, D. V., The Laplace transform, Princeton University Press, 1946.

DEPARTMENT OF MATHEMATICS

41296 GÖTEBORG

SWEDEN 